\title{
A New Performance Index for Evaluating Transit Quality of Service
}

\author{
Liping Fu, University of Waterloo, Waterloo, Ontario, Canada \\ Yaping Xin, Stantec Consulting Ltd., Edmonton, Alberta, Canada
}

\begin{abstract}
This article proposes a new performance index called Transit Service Indicator (TSI), which could be used as a comprehensive measure for quantifying the quality of service of a transit system. TSI integrates multiple performance measures (e.g., service frequency, hours of service, route coverage, and travel time components) within a systematic framework. It takes into account spatial and temporal variations in travel demand, recognizing that quality of service is a result of interaction between supply and demand. A case study is conducted to examine the sensitivity of the proposed TSI to various system design and condition variables and parameters.
\end{abstract}

\section{Introduction}

The latest Transit Capacity and Quality of Service Manual (TCQSM; Kittelson \& Associates 2003 ) is an excellent supplement to the widely accepted Highway Capacity Manual (HCM; Transportation Research Board 2000), with a systematic framework for addressing various conceptual and methodological issues related to transit capacity analysis and quality-of-service evaluation. One of the main features of the TCQSM is its adoption of the level-of-service (LOS) concept introduced in the HCM for measuring the quality of transit service from users' perspectives. 
As acknowledged in the TCQSM, quantifying the quality of service of a transit system is much more complicated than evaluating a highway facility because of the involvement of multiple players (e.g., transit operators, passengers, vehicles) and a wide range of interrelated factors (e.g., spatial and temporal coverage, comfort level, reliability). As a result, the current TCQSM has opted to use multiple LOS measures, instead of one or two measures as the HCM, to evaluate the quality of service of a transit system or its specific components. For example, six LOS measures are proposed for evaluating the quality of service of a fixed-route transit system, encompassing both service availability (service frequency, service span, service coverage) and service quality (passenger loading, service reliability, transitauto travel time difference). These measures reflect different aspects of a transit service system as perceived by typical transit users.

One of the major disadvantages of using multiple LOS measures is its difficulty to provide an overall quality-of-service evaluation required for comparing different transit routes, travel corridors, or transit systems. The main objective of this research is to explore the possibility of combining some of the LOS measures into a single quality-of-service measure.

The attempt to develop a comprehensive quality-of-service index is not new. Several past studies have made considerable progress on developing service indices to measure transit quality of service, as summarized in Table 1. Rood (1997) proposed a service availability measure called Local Index of Transit Availability (LITA), which includes three components: frequency, capacity, and route coverage. Hillman (1997) developed the Public Transportation Accessibility Level (PTAL) index to measure the access availability to the public transit network. Florida DOT introduced a new quality-of-service measure called Transit Level of Service indicator (TLOS), which is defined as the percentage of time that an average person can use the transit service (Kittelson \& Associates and URS, Inc. 2001). This indicator incorporates the coverage, frequency, duration of the transit service, availability and quality of pedestrian paths to transit stops, as well as the number of people and jobs receiving transit service. All these indices model only the availability aspect of a transit system with no consideration on service convenience (e.g., travel time) and demand distribution.

Polzin et al. (2002) were the first to suggest the need to consider demand distribution in evaluating transit quality of service. They proposed the Transit Service Accessibility Index (TSAI), which measures how well travel demand is served using time-of-day travel demand distribution to determine the relative value of the 


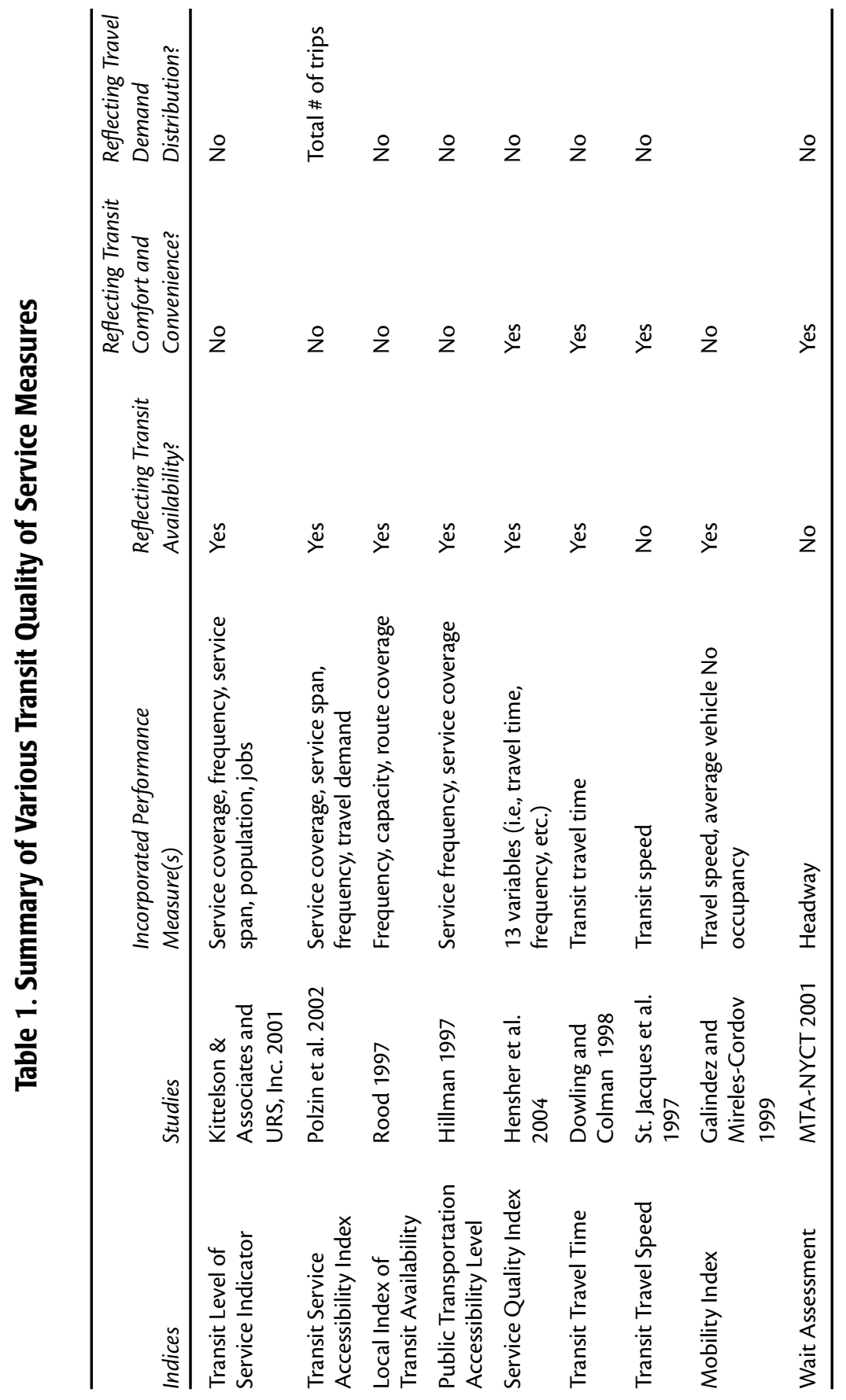


transit service provided in each time period of the day. The travel demand distribution considered is, however, limited to temporal fluctuation along the transit route and no spatial distribution is considered.

Galindez and Mireles-Cordov (1997) developed a mobility index for the Los Angeles County Metropolitan Transportation Authority, which is defined as the production of average travel speed and average vehicle occupancy. However, this index considers only travel convenience (travel time and comfort), overlooking other quality-of-service aspects such as coverage, frequency, and service span. The Service Quality Index (SQI) proposed by Hensher et al. (2004) adopts a stated choice (SC) method, in which a sample of passengers were asked to choose their most preferred travel option from a number of alternatives with known attributes. Multinomial logit (MNL) models were then estimated to obtain the relative weights representing the contribution of each service attribute to the preference of travelers. The resulting weights were used in calculating the overall SQI of a transit system. This model reflects an individual's view of a transit system in general. However, it is not clear how the locally calibrated model can be applied to transit systems in other geographical areas. Furthermore, an aggregation scheme needs to be developed to obtain the collective view of all potential users on a given transit system.

A new transit quality-of-service index, called Transit Service Indicator (TSI), is introduced in this article. The study presents a detailed computational procedure that implements the proposed methodology, and performs a sensitivity analysis to evaluate the relationship between the proposed TSI and various system characteristics.

\section{Methodology}

\section{Transit Service Indicator from a Single Trip-Maker's Perspective}

The starting point of our proposed methodology is to address the question of how to measure the quality of service of a transit system from a given trip-maker's point of view. In this research, we contemplate that the perception of a trip-maker on a transit system can be mostly reflected by his or her perceived total travel time by transit as compared to auto travel time for the trip. As a result, we propose to use the ratio of the weighted door-to-door travel time by auto (WTA) to the weighted door-to-door travel time by transit (WTT) as a performance indicator, called Transit Service Indicator (TSI), to measure the quality of service for a given 
trip of a given individual. Specifically, the TSI for a trip from origin point $i$ to destination point $j$ at time period $t$ is defined as follows:

$$
\operatorname{TSI}(i, j, t)=\frac{W T A(i, j, t)}{W T T(i, j, t)}
$$

where:

$W T A(i, j, t) \quad$ is the weighted total of driving time and walk time from origin $i$ to destination $j$ at time period $t$ by auto

$W T T(i, j, t) \quad$ is the weighted transit travel time from the same origin point $i$ to the same destination point $j$ at time period $t$

A detailed discussion on these two travel times and their components is provided later in this article.

\section{Transit Service Indicator from Multiple Trip-Makers' Perspectives}

The transit service indicator introduced in the previous section can be used to represent the quality of service of a transit system from a given individual's perspective. From a practical point of view, however, a measure of quality of transit service should reflect the collective view of all the individuals who are covered by the transit system. To measure such a collective view, we must ideally consider both the temporal and spatial variations of the individual trips (demand) and the availability of transit service to serve these trips. In this section, we propose a simulation-based methodology that can be used to achieve this objective. We first discuss how to measure the transit service indicator along a travel corridor, which is then extended to the case of a given activity area, and further to a whole service area.

TSI of a Travel Corridor. A travel corridor linking two activity areas is shown in Figure 1, where $A O$ (Area of Origins) represents an area where all trips start and $\mathrm{AD}$ (Area of Destinations) represents an area where all trips end. An activity area usually refers to an area with high-density population or employment, which could be a transportation analysis zone (TAZ) or a combination of several such zones. Without loss of generality, we will consider only one-way trips, assuming that total daily trips (demand) and transit service (supply) between the areas are symmetrical and balanced. The activity areas are connected by a network of streets and transit routes. To determine the "total" view of all trip-makers who 


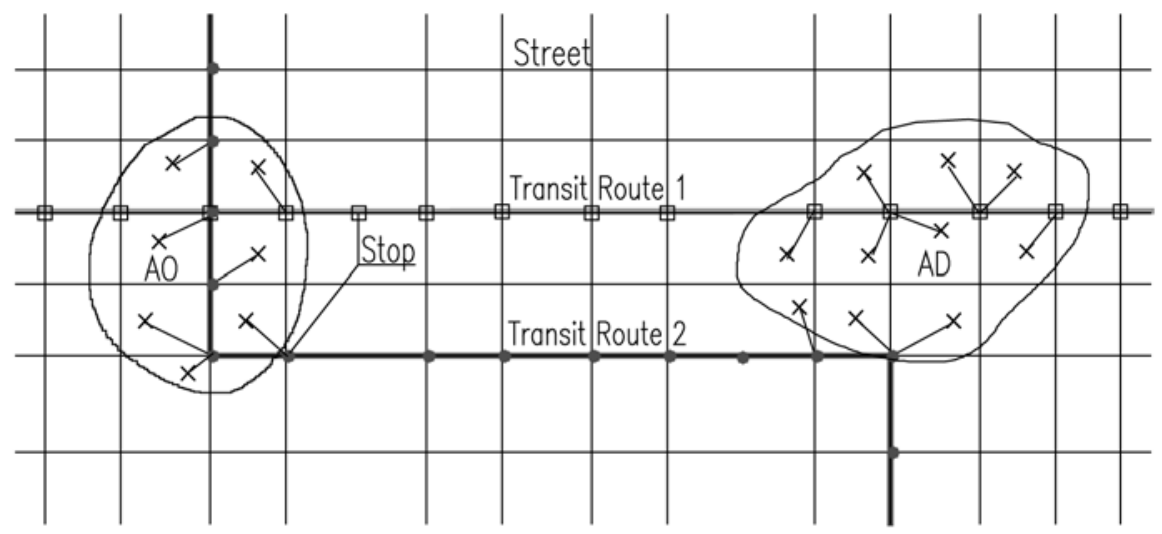

Figure 1. A Travel Corridor Between Two Activity Areas

travel from $A O$ to $A D$, a set of trip origins and destinations is randomly generated on the basis of the activity distribution pattern of each area, and the transit quality index for each trip (with known origin and destination) is subsequently calculated using Equation (1).

Depending on the actual location of the trip origin and destination, different trips could yield different travel paths and thus different TSI values. The idea behind the proposed method is using the average TSI value of a set of randomly generated trips to represent the combined perspective of all travelers along the corridor. If a total of $\mathrm{nAO}$ random origin points $(i)$ and a total of $\mathrm{n}_{\mathrm{AD}}$ random destination points $(j)$ are considered for time period $t$, a total of $\mathrm{n}_{\mathrm{AO}} \times \mathrm{n}_{\mathrm{AD}} \mathrm{O}$-D pairs can be formed and the average TSI between $\mathrm{AO}$ and $\mathrm{AD}$ for the period can therefore be expressed as follows:

$$
\operatorname{TSI}(A O, A D, t)=\frac{\sum_{i=1}^{n_{A O}} \sum_{j=1}^{n_{A D}} \operatorname{TSI}(i, j, t)}{n_{A O} * n_{A D}}
$$

where:

$n_{A O}, n_{A D} \quad$ equals number of points randomly generated in area $\mathrm{AO}$ and $A D$, respectively 
If the total demand from $\mathrm{AO}$ to $\mathrm{AD}$ in time period $t$ is denoted as $T O D(A O, A D$, $t$ ), the overall average TSI along the travel corridor in a whole day can be obtained using Equation (3).

$$
\operatorname{TSI}(A O, A D)=\frac{\sum_{t=1}^{n_{t}} \operatorname{TSI}(A O, A D, t) * \operatorname{TOD}(A O, A D, t)}{\sum_{t=1}^{n_{t}} \operatorname{TOD}(A O, A D, t)}
$$

where:

$\operatorname{TSI}(A O, A D)$ equals daily $\mathrm{TSI}$ value for the travel corridor from $\mathrm{AO}$ to $\mathrm{AD}$ $\operatorname{TOD}(A O, A D, t)$ represents the total travel demand from $\mathrm{AO}$ to $\mathrm{AD}$ at time period

$n_{t} \quad$ is the number of time periods

TSI of an Activity Area. The quality of transit service of a given activity area is defined as the combined quality of transit service from that area to all desired destination areas. As a result, the TSI of a given area for a given period can be formulated as follows:

$$
\operatorname{TSI}(A O, t)=\frac{\sum_{A D \in Z_{D}} \operatorname{TSI}(A O, A D, t) * \operatorname{TOD}(A O, A D, t)}{\sum_{A D \in Z_{D}} \operatorname{TOD}(A O, A D, t)}
$$

where:

$\operatorname{TSI}(A O, t)$ equals TSI value for area $\mathrm{AO}$ at time period $t$

$Z_{D} \quad$ is the set of destination areas 
Equation (4) can be further integrated to obtain the daily average TSI for a given activity area as follows:

$$
\operatorname{TSI}(A O)=\frac{\sum_{t=1}^{n_{t}} \operatorname{TSI}(A O, t) * \operatorname{TOD}(A O, t)}{\sum_{t=1}^{n_{t}} \operatorname{TOD}(A O, t)}
$$

where:

$\operatorname{TSI}(A O) \quad$ is the daily TSI value for the given area AO

$\operatorname{TOD}(A O, t) \quad$ equals total travel demand originating from the area $\mathrm{AO}$ at time period $t$, which can be calculated as follows:

$$
T O D(A O, t)=\sum_{A D \in Z_{D}} T O D(A O, A D, t)
$$

TSI of a Service Area. Following the same idea, we can formulate the TSI for the whole service area covered by a transit system as follows:

$$
\begin{aligned}
& T S I(t)=\frac{\sum_{A O \in Z_{O}} \operatorname{TSI}(A O, t)^{*} \operatorname{TOD}(A O, t)}{\sum_{A O \in Z_{O}} T O D(A O, t)} \\
& T S I=\frac{\sum_{t=1}^{n_{t}} T S I(t)^{*} \operatorname{TOD}(t)}{\sum_{t=1}^{n_{t}} \operatorname{TOD}(t)}
\end{aligned}
$$

where:

$\operatorname{TSI}(t) \quad$ equals TSI value for a service area at time period $t$

TSI is the daily TSI value for a transit service area 
$\operatorname{TOD}(t)$ represents total demand from all origins to all destinations in time period $t$, which can be determined as follows:

$$
T O D(t)=\sum_{A O \in Z_{O}} \sum_{A D \in Z_{D}} T O D(A O, A D, t)
$$

where:

$\mathrm{Z}_{\mathrm{O}} \quad$ is the set of origin areas within the service area

$Z_{D} \quad$ equals the set of destination areas within the service area

The TSI value defined by Equations (3-8) uses total travel demand instead of transit demand as a weighting factor to aggregate users' points of view, which assumes all trip-makers are potential transit users. This assumption could be relaxed in practice by considering only transit demand.

\section{Estimation of Auto/Transit Travel Times}

Since the proposed transit service indicator is defined on the basis of travel time by both transit and auto mode as shown in Equation (1), it is necessary to develop an accurate estimate of the expected travel time for each trip by each mode. Figure 2 shows the procedure involved in estimating auto/transit travel times. As can be seen, travel time calculation is not straightforward due to the existence of multiple paths for a given trip, the interaction between travel time (supply) and

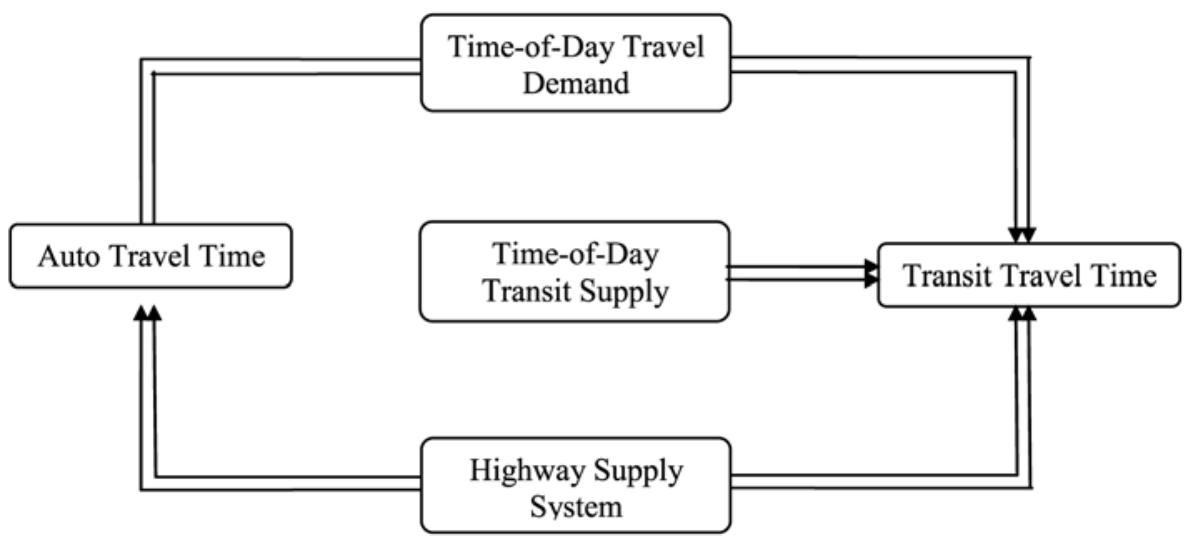

Figure 2. Estimation of Auto/Transit Travel Times under Congested Conditions 
traffic/passenger volume (demand), and variation in trip-making behaviors. Ideally, the paths that are actually being taken or are considered as valid alternatives by the trip-maker should be identified and used in the calculation. In this research, we made the following assumptions to limit the complexity of the analysis:

- All trip-makers, using either transit or auto, are assumed to prefer the path that has the lowest weighted travel time, or the best path.

- The headways of transit routes in each service period are assumed constant and the average passenger wait time is half of the service headway. For routes with large service headway (e.g., more than 15 minutes), transit users could time their arrival at the stop according to the published schedule and thus experience a waiting time of less than half of the headway. However, from the point of view of service availability, a passenger would still have to wait for half of the headway on average before being able to use the service. A certain portion of the waiting time, however, could be spent at home or office if a schedule is followed.

- Access and egress times for auto users are negligible.

Auto Travel Time. Auto travelers usually have a choice of many alternative paths to their destinations. For each path, the door-to-door auto travel time (TA) can be obtained by summing up the time on all traveled links, which is given in the following equation:

$$
T A_{k}(i, j, t)=\sum_{a \in k} \frac{d_{a}(i, j, t)}{S A_{a}(i, j, t)}
$$

where:

$T A_{k}(i, j, t) \quad$ equals auto travel time along path $k$ from origin $i$ to destination $j$ at time period $t$

$a$ is the link number for the auto-traveled path $k$

$d a(i, j, t) \quad$ shows distance of link a for the $i-j$ trip at the time period $t$

$S A_{a}(i, j, t) \quad$ is average auto travel speed on link $a$ for the $i-j$ trip at time period $t$, which depends on traffic volume of the link 
Since the access and egress times for auto travel are not taken into account, the weighted door-to-door auto travel time (WTA) for a given trip $i$ - $j$ at time period $t$ can therefore be determined by identifying the path with the lowest weighted auto in-vehicle travel time using a shortest path algorithm, that is

$$
W T A(i, j, t)=\operatorname{Min}\left[T A_{k}(i, j, t)\right]
$$

Because of the dependency of link travel speed on traffic volume, travel speeds on individual links should be obtained either from field observations or a traffic prediction model that simulates user-equilibrium (UE) traffic conditions (Sheffi 1984).

Transit Travel Time. Total door-to-door transit travel time for a given transit itinerary is determined by combining the different travel time components as follows:

$$
\begin{aligned}
& T T(i, j, t)=T T^{L}(i, j, t)+T T^{W k}(i, j, t)+T T^{W t}(i, j, t) \\
& T T^{L}(i, j, t)=\sum_{b} \frac{d_{b}(i, j, t)}{S T_{b}(i, j, t)} \\
& T T^{W k}(i, j, t)=\left[d^{A s}(i, j, t)+d^{E s}(i, j, t)+d^{T s}(i, j, t)\right] / S W \\
& T T^{W t}(i, j, t)=\sum_{m=1}^{T r} \frac{h_{m}(i, j, t)}{2}
\end{aligned}
$$

where:

$T T(i, j, t) \quad$ is door-to-door transit travel time from origin $i$ to destination $j$ at time period $t$

$T T^{L}(i, j, t) \quad$ represents transit line-haul time from origin $i$ to destination $j$ at time period $t$

$T T^{W k}(i, j, t) \quad$ equals total walk time for transit mode from origin $i$ to destination $j$ at time period $t$, including access time, egress time, and transfer walk time, if applicable 
$T T^{W t}(i, j, t) \quad$ is total wait time for transit mode from origin $i$ to destination $j$ at time period $t$, including initial wait time and transfer wait time, if applicable

$b \quad$ equals the link number for the transit itinerary

$d_{b}(i, j, t) \quad$ represents distance of link $b$ for trip $i$-j at time period $t$

$S T_{b}(i, j, t) \quad$ shows transit travel speed on link $b$ for the $i$-j trip at time period $t$ $d^{A s}(i, j, t), d^{E s}(i, j, t), d^{T s}(i, j, t)$ is the distance for access link(s), egress link(s), and transfer link(s) respectively for trip $i-j$ at time period $t$

SW equals walking speed (e.g., $5 \mathrm{~km} / \mathrm{h}$ )

$h_{m}(i, j, t) \quad$ represents headway of transit route $m$ for trip $i$-j at time period $t$

$\operatorname{Tr} \quad$ is the number of transit routes required for trip $i-j$ at time period $t$

The weighted door-to-door transit travel time from origin $i$ to destination $j$ at the $\mathrm{t}^{\text {th }}$ time period, WTT $(\mathrm{i}, \mathrm{j}, \mathrm{t})$, can then be obtained by multiplying the weighting factors to the corresponding travel time components.

$$
W T T(i, j, t)=f^{W k} * T T^{W k}(i, j, t)+f^{W t} * T T^{W t}(i, j, t)+f^{L} * T T^{L}(i, j, t)
$$

where:

$f^{W k}, f^{W t}, f^{L} \quad$ equals weighting factors for walk time, wait time, and line-haul time, respectively

Finding the best path in a transit network could become more complicated than in a road network, because passengers are more likely to make adaptive decisions on which route to take at the starting or transferring point, depending on the time they arrive at the stop and the availability of transit service after their arrival. The shortest path algorithm can be used to find the best path from the multiple paths; however, it assumes that only one fixed transit line will be chosen, which is not a realistic representation as pointed by Spiess and Florian (1989). They proposed a new methodology called Optimal Strategy Method (OSM), which models that passengers may board different routes at the same stop to reach the destination. Due to its more reasonable assumption, OSM has been made available in many transportation planning software packages, such as EMME/2 (INRO Consultants) 
and TransCAD (Caliper Corporation). In the following analysis, we also use OSM to determine the weighted transit travel time.

A number of studies have been conducted to quantify the differences in passengers' perceptions on different travel time components. Generally, it has been suggested that "out-of-vehicle time," which includes wait time, transfer time, and walk time, is at least twice as important as "in-vehicle time" (Quarmby 1967; Shunk and Bouchard 1970; Schultz 1991). As summarized by Pratt (2000), weighting factors for transit travel time vary by location and trip purposes, and therefore should be determined on the basis of local conditions. Also, the concept of travel time weighting factors are closely related to the concept of utility functions used in logit mode choice modeling from which the values for the weighting factors can be directly obtained (Hensher et al. 2004).

\section{Computational Procedure}

The proposed quality-of-service evaluation method requires extensive input data and processing, and is therefore best done through a computer program. This section describes the steps involved in determining the TSI for a given travel corridor. (Note that similar steps are involved for different analysis scopes such as a given area or a city.) This procedure has been implemented and tested using TransCAD with the steps outlined below.

\section{Step 0: Prepare Input Data}

This step prepares all the data required for TSI analysis, including:

Street Network Data. A street network consists of a set of nodes and links. Nodes are identified by longitude and latitude coordinates, while links are associated with a number of attributes, such as length, speed limit, transit speed, and walk speed. The speed that a transit vehicle will operate on a street is also stored as a link attribute. Since transit speed depends not only on the street condition and traffic congestion, but also on the stop spacing and location (online or offline), fare collection, and passenger demand, it is therefore quite complicated to decide the transit speed. The TCQSM recommends that the best way to obtain transit speeds is to measure them in the field directly. For an existing transit system, we can also check the transit schedules to determine the average speed assumed in transit planning. Walking speed is usually assumed to be $5 \mathrm{~km} /$ hour. To predict travel time under congested conditions, travel time functions associated with individual links must also be provided. For example, if the commonly used Bureau of Public 
Roads (BPR) travel time function is used, the associated parameters need to be specified for each type of link.

Transit Network Data. Transit network data include attributes on transit routes and stops. All the stops, including terminals (or stations), time points, and regular stops, should be prepared. Transit routes are usually built upon the underlying street network with route segments associated with road network links and stops located at nodes or links. Associated with each transit route are attributes, including route ID, route headway, route weight (used to distinguish local bus from express bus), etc. Transit stops are identified by longitude and latitude, a milepost to indicate the route direction, and a route ID that provides a reference between stops and routes. Further, service for different time periods could be managed separately using different datasets. These data may be obtained from the local transit agency.

Weighting Factors for Travel Time Components. Weighting factors can usually be determined by planners based on local conditions or results from other studies.

Demographic and Employment Profile. To identify the activity areas associated with a transit system, both demographic and employment data on a zonal basis are needed. These data are usually available from the local transportation planning department, including zonal boundary information, centroids, population and household data, employment data, etc.

Travel Demand Data. The O-D matrices of auto trips and transit trips for each analysis period should be obtained. Most transportation planning departments only have peak-hour auto and transit O-D travel demand. Travel demand in other time periods could be obtained using typical hourly traffic variation factors or through a specific demand estimation process (Institute of Traffic Engineers 1992).

\section{Step 1: Identify Activity Areas Along the Travel Corridor under Evaluation}

This step identifies areas that have high population and employment density and that contribute significantly to the traffic along the corridor. An activity area can consist of a single TAZ or a combination of several TAZs.

\section{Step 2: Generate Trip Ends (origins and destinations)}

A set of trip ends is randomly generated on the basis of area characteristics (e.g., for trip origins, residential density could be used). The minimum number of trip ends that needs to be generated depends mainly on the size of the activity areas. A minimum of 30 trips or 5 to 6 trip ends in each area is recommended. 


\section{Step 3: Connect Trip Ends with the Street Network}

The randomly generated trip ends are then connected to the street network by linking them to the closest nodes. This is necessary for a computer program to search for the shortest paths between the trip ends. To make the representation more realistic, two connectors for each trip end could be used. Figure 3 gives an example of randomly generated trip ends and their connections to the network.

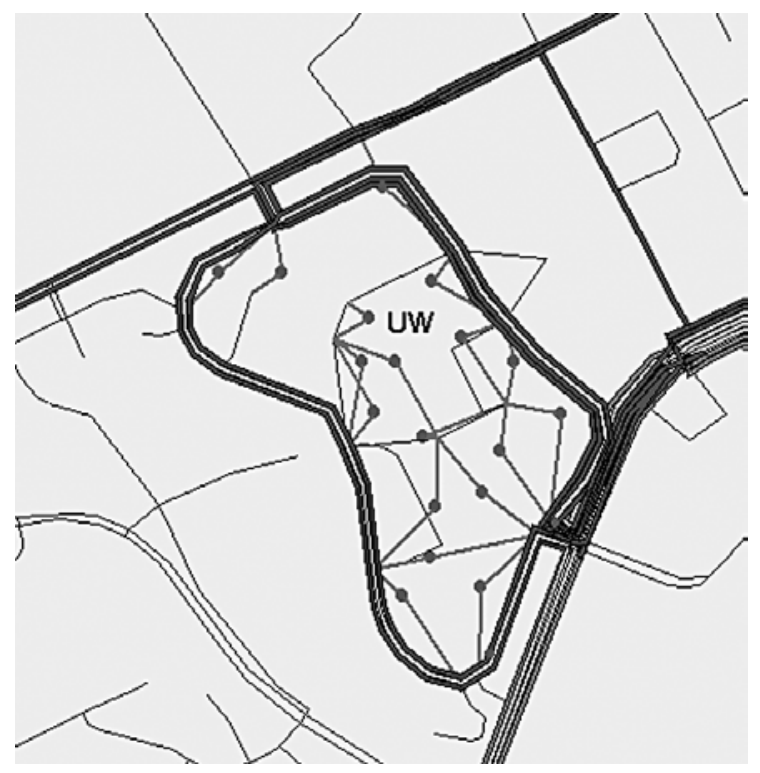

Figure 3. Example of Random Trip Ends and Connections

\section{Step 4: Perform Traffic Assignment}

Use a user equilibrium method to assign total auto travel demand to the street network, which will yield traffic volume and auto travel time on each link. The auto travel time field in the street network database can then be updated with the equilibrium travel time.

\section{Step 5: Calculate WTA}

Use a shortest path algorithm to calculate the auto travel time for each trip. In TransCAD, we can use the Network/Paths/Multiple Paths function to calculate the shortest auto travel times between multiple points automatically. 


\section{Step 6: Calculate WTT}

This step determines the transit travel time for each trip based on the Optimal Strategy Method described previously. TransCAD's Transit/Multiple Paths function calculates the weighted door-to-door transit travel times among multiple points.

\section{Step 7: Calculate TSI}

With WTA and WTT, we can calculate the TSI along the travel corridor using Equations (1-3).

\section{Sensitivity Analysis}

A key indicator for evaluating the suitability of a LOS measure for a transit system is its sensitivity to policy and design variables, such as headway, service hours, and spatial coverage. This section describes a sensitivity analysis of the proposed TSI by applying it to a realistic travel corridor under a set of hypothetical service design options. As shown in Figure 4, the travel corridor selected for this analysis was extracted from the Region of Waterloo, Ontario, consisting of three activity centers: Kitchener Transportation Center (KTC), Kitchener South West Residential area (KSWR), and KSWR_CBD, where KSWR_CBD is a subarea of KSWR and is introduced for the purpose of evaluating the impacts of spatial coverage. To control the scope of the analysis, only one route was considered (Route \#2), which connects the three activity centers. The headway for this route is 30 minutes in AM peak period, midday, and PM peak period, and 45 minutes in the evening period (The Region of Waterloo 2004).

\section{Sensitivity to Spatial Availability of Transit Service}

To evaluate the sensitivity of the proposed TSI to the spatial coverage of a transit system, we compare two travel corridors: KTC-KSWR and KTC-KSWR_CBD, as shown in Figure 4. The figure also shows the area that is covered by transit service using 400 meters as the reasonable walking distance from transit stops. Based on the LOS evaluation method suggested by the TCQSM, transit coverage for KSWR CBD is almost the same as KSWR, while it is clear that trips ending at KSWR_CBD have a much better distance coverage than trips ending at KSWR. The proposed TSI takes into account detailed spatial distribution of trip ends and can thus reveal such subtle difference. Figure 5 shows the TSI values of the two scenarios for the PM peak and evening period under free-flow traffic conditions (i.e., traffic congestion is not considered in estimating auto and transit travel times). As shown, the 


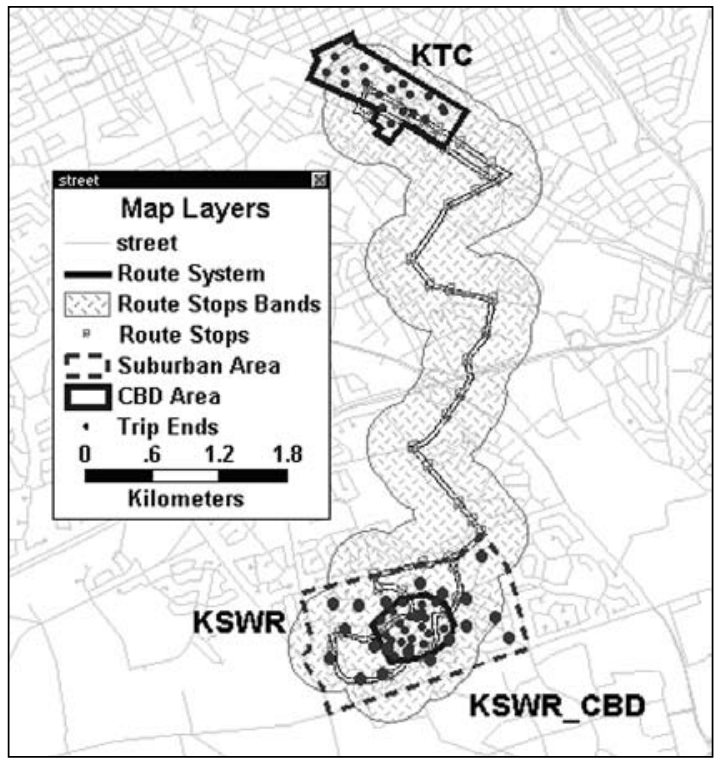

Figure 4. A Travel Corridor for Sensitivity Analysis (Route \#2)

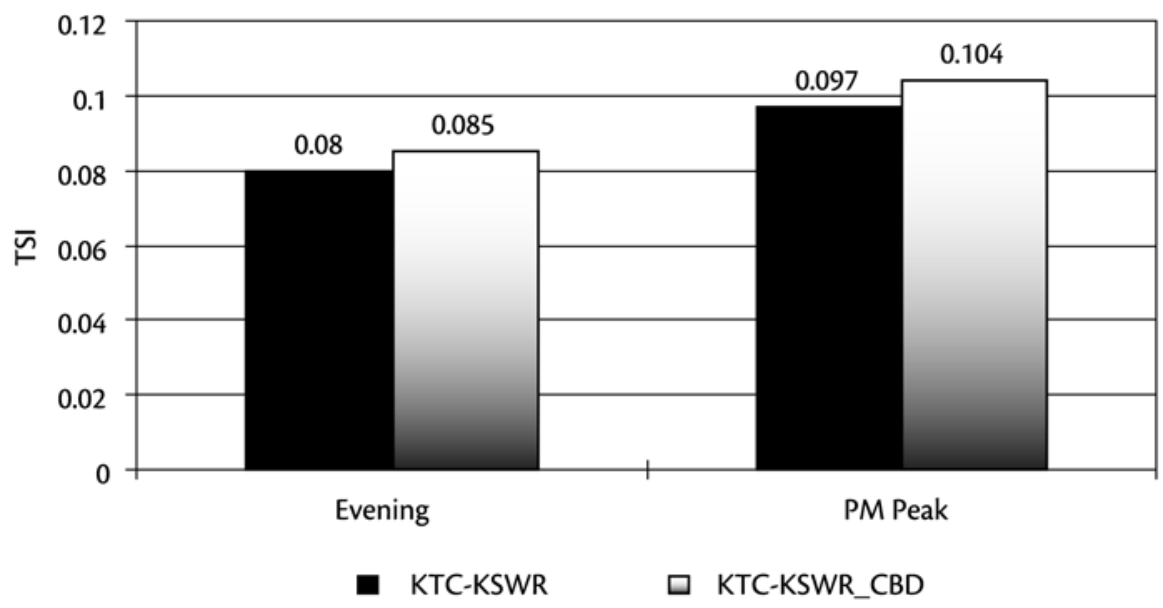

Figure 5. Sensitivity to Spatial Availability 
TSI value of the KSWR_CBD area is about 7 percent higher than the TSI of the larger area-KSWR for both peak and off-peak periods, due to a better service coverage in the CBD area.

\section{Sensitivity to Temporal Availability of Transit Service}

As described previously, the travel corridor is served by the transit route with a headway of 30 minutes in PM peak period and 45 minutes in the evening. As a result, a comparison of TSI between these time periods can reveal the sensitivity of the proposed TSI to temporal availability of transit service. Figure 6 shows the TSI for the two assumed travel pairs in two different time periods (again under free-flow traffic conditions). As expected, the higher the service frequency, the higher the TSI value and the level of service. The difference in TSI value between the PM PK period and the evening period is approximately 20 percent. This difference is not in proportion to the difference in the corresponding service frequency (50\%). One of the interpretations of this result could be that a reduction in service frequency would not translate to the same amount of reduction in the quality of transit service.

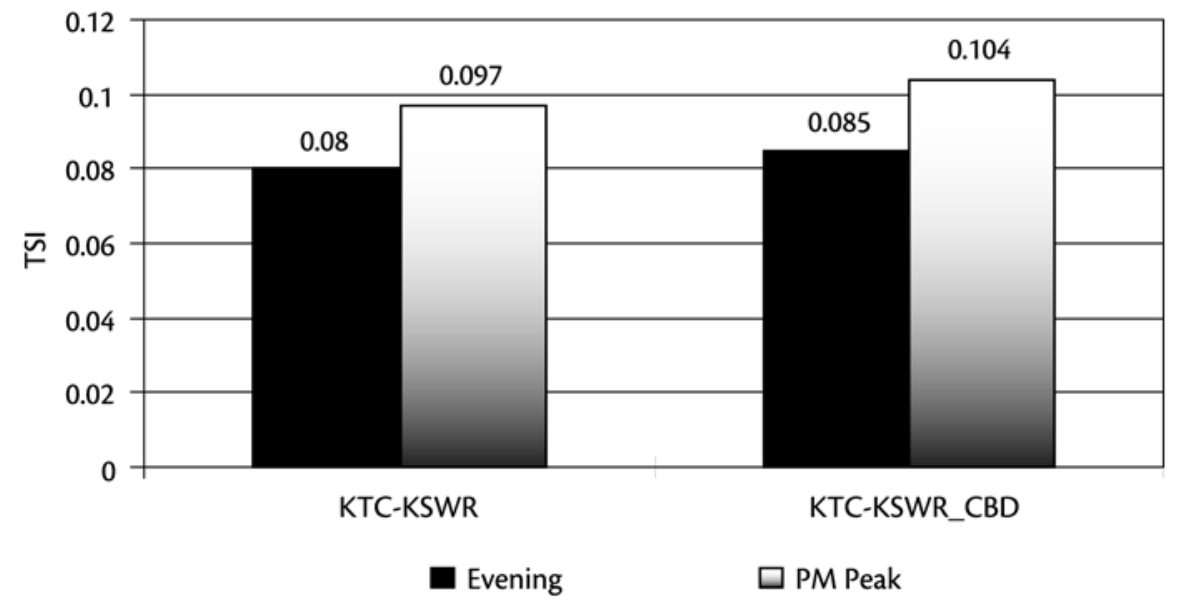

Figure 6. Sensitivity to Service Frequnecy

\section{Sensitivity to Travel Demand Variation}

The daily TSI defined in this research takes into account travel demand variation over time. As a result, different travel demand distributions would result in different TSI values under the same transit service, which would otherwise not be 
revealed using the existing LOS approach. To evaluate this relationship, we created three variations of travel demand on the travel corridor (Figure 7): 1 constant demand, normal demand variation, and high demand variation. With the assumed time-of-day demand variation, we can calculate the daily TSI of the corridor from KTC to KSWR under the same transit service route and schedule.

The results in Figure 8 indicate that daily TSI is highly sensitive to temporal variation of travel demand. An approximate 14 percent difference in TSI was observed between the constant demand case and high demand variation case. This suggests that the proposed TSI has the attribute of reflecting the degree of match (or

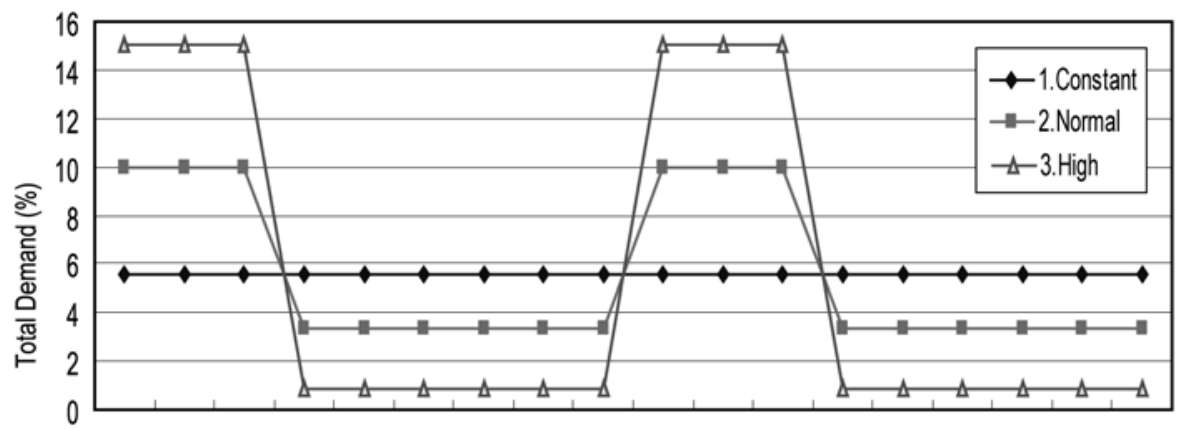

0600-0700-0800-0900-1000-1100-1200-1300-1400-1500-1600-1700-1800-1900-2000-2100-2200-2300$07000800 \quad 0900100011001200130014001500 \quad 1600 \quad 170018001900 \quad 20002100 \quad 2200 \quad 23002400$

Time Period

Figure 7. Time-of-Day Travel Demand Variation

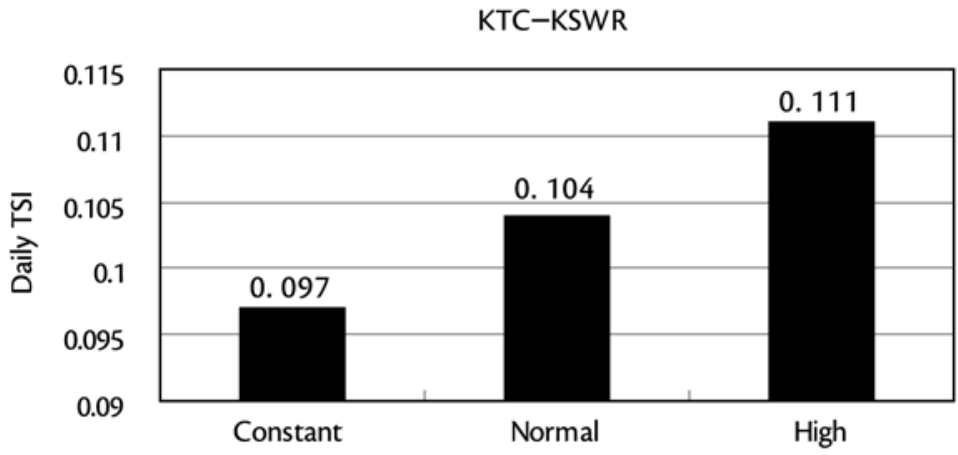

Time-of-Day Demand Variations

Figure 8. Sensitivity to Travel Demand Variation 
mismatch) between demand and supply (service frequency). As a result, it may be used to guide the allocation of resources (vehicles and service frequency) among different service periods.

\section{Sensitivity to Traffic Congestion}

The proposed TSI considers the quality of travel by auto as a basis in defining the quality of service of transit travel, and should therefore be dependent on traffic congestion. To evaluate the dependency of TSI on traffic congestion, we investigated two scenarios. The first scenario considers free-flow traffic conditions; that is, travel demand was not assigned to network when calculating auto/transit travel time. In the second case, we first assigned auto travel demand to the road network by user equilibrium method, and auto/transit travel times were then calculated. Figure 9 shows the TSI for a travel corridor with and without considering traffic congestion in two different time periods. As shown, the proposed TSI is quite sensitive to traffic congestion. The higher the traffic congestion, the higher the TSI value or the higher the quality of transit service as compared to auto travel.

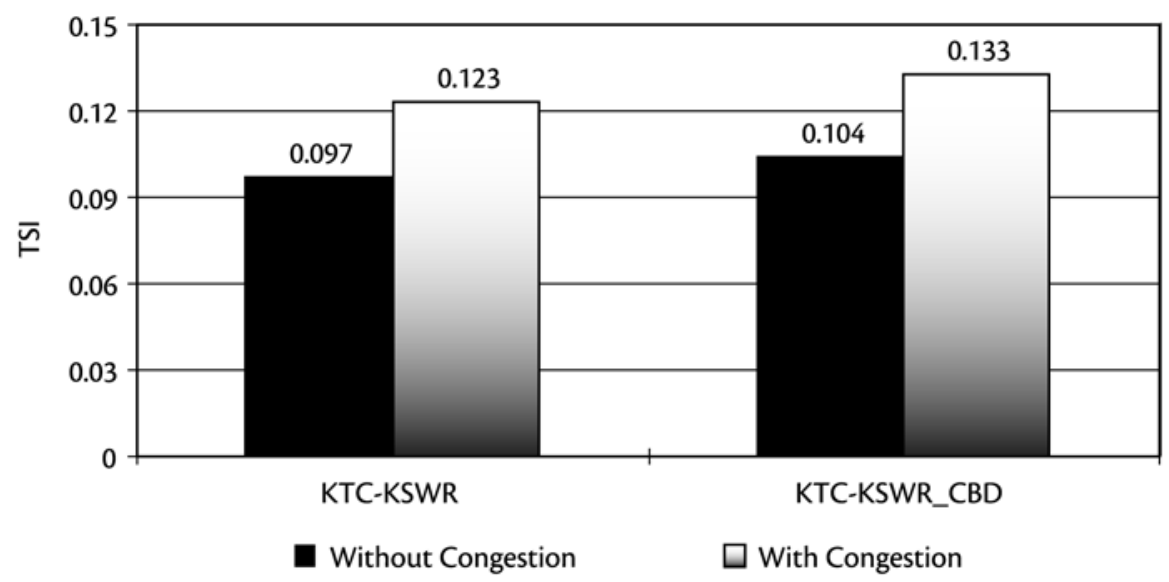

Figure 9. Sensitivity to Traffic Congestion

\section{Conclusions}

This research introduced the Transit Service Indicator (TSI), a new performance index that can be used as a comprehensive measure for evaluating the quality of service of a transit system. Different from existing transit performance indices, the 
proposed TSI integrates a number of performance measures with vastly different natures, such as service headway, service hours, route coverage, and various travel time components (walk, wait, transfer, and ride). Furthermore, it recognizes the need to account for the effects of both supply and demand with a LOS measure that includes demand as a part of the equation. A sensitivity analysis on the effects of service coverage, headway, demand distribution, and traffic congestion has indicated that the proposed TSI is sensitive to various design and condition variables and has the potential to be used as a replacement of or supplement to some existing LOS measures.

This research is limited in the following aspects:

- The proposed TSI considers travel time as the dominant factor influencing travelers' views on transit quality of service. This assumption may not hold in many complex travel environments. Other factors such as comfort level in a transit vehicle, out-of-pocket costs, parking availability and costs, safety, and reliability all could be important in users' views on the quality of service of a transit system. Future research should examine the possibility of using generalized cost instead of weighted travel time to define the transit service index.

- Based on the proposed TSI, the quality of transit service would improve as highway congestion increases (even though the transit service remains the same). Further investigation is required to examine the desirability of such dependency. Also, applicability of other types of measures such time difference and relative time difference should be investigated.

- The proposed analysis methodology requires some cumbersome calculation and significant amount of data (e.g., road and transit network). Custom programs that can be added to specific GIS tools should be developed to automate the calculations.

- This research did not make any attempt to define LOS using the proposed TSI. Future research should therefore explore the possibility of establishing a mapping between TSI values and various LOS scales (A-F) that are used in the TCQSM. This would require a clear definition of TSI values that represent acceptable or unacceptable services, which is only possible through a survey of transit operators, planners, and passengers.

- Finally, this research could be further enhanced with a sensitivity analysis that covers a wide range of case applications featuring different property 
sizes, operating environments, and system characteristics. Such an analysis is necessary before the conclusions obtained in this study can be generalized.

\section{Acknowledgments}

This research was supported by the Natural Sciences and Engineering Research Council of Canada. We would like to thank the Region of Waterloo, especially Mr. Dave Durant, for kindly providing data for this research.

\section{References}

Galindez, A. A., and R. Mireles-Cordov. 1999. Visualization of transit mobility and performance. Presented at 78th Annual Meeting of the Transportation Research Board, Washington, DC.

Hensher D., P. Stopher, and P. Bullok. 2004. Service quality-Developing a service quality index (SQI) in the provision of commercial bus contracts. http://www. its.usyd.edu.au/bus_and_coach_themes/SQI4paper_Sep01.pdf. Accessed May 2004.

Hillman, R., and G. Pool. 1997. GIS-based Innovations for modeling public transport accessibility. Traffic Engineering and Control 38 (10): 554-559.

Institute of Traffic Engineers. 1992. Traffic engineering handbook, 4th ed.

Kittelson \& Associates, Inc. 2003. Transit capacity and quality of service manual, 2nd ed. TCRP Project 100. Washington, DC: TRB, National Research Council. http://trb.org/publications/tcrp/tcrp100. Accessed March 2004.

Kittelson \& Associates, Inc., and URS, Inc. 2001. Transit level of service (TLOS) software user's guide. Version 3.1. Developed for Florida Department of Transportation Public Transit Office.

Polzin, S., R. Pendyala, and S. Navari. 2002. Development of time-of-day-based transit accessibility analysis tool. Transportation Research Record: Journal of Transportation Research Board 1799. Washington, DC: TRB, National Research Council, 35-41.

Pratt, R. H. 2000. TCRP Web document 12: Traveler response to transportation systems changes: Interim handbook. Washington, DC: TRB, National Research 
Council. http://gulliver.trb.org/publications/tcrp/tcrp_webdoc_12.pdf. Accessed May 2004.

Quarmby, D. A. 1967. Choice of travel mode for the journey to work-some findings. Journal of Transport Economics and Policy 1 (3).

The Region of Waterloo. 2004. Grand River Transit (GRT) web documents. http:// www.grt.ca. Accessed March 2004.

Rood, Timothy. 1997. Local index of transit availability: Riverside County, California case study report. Sacramento: Local Government Commission.

Schultz, G. W. 1991. Modeling approach. Memorandum to Seattle Metro Files.

Sheffi, Y. 1984. Urban transportation networks: Equilibrium analysis with mathematical programming methods. Englewood Cliffs, NJ: Prentice-Hall.

Shunk, G. A., and R. J. Bouchard. 1970. An application of marginal utility to travel mode choice. Highway Research Record No. 322.

Spiess, H., and M. Florian. 1989. Optimal strategies: A new assignment model for transit networks. Transportation Research B 23B (2): 83-102.

Transportation Research Board. 2000. Highway capacity manual. Washington, DC: National Research Council.

LIPING Fu (Ifu@uwaterloo.ca) is an associate professor in the Department of Civil Engineering at the University of Waterloo, Ontario, Canada. Dr. Fu's research interest focuses on evaluation and optimization of large, complex traffic and transportation service systems and on the development of decision support tools for use in managing these systems. Dr. Fu has contributed to research in the areas of intelligent transportation systems, public transit, traffic safety, and winter road maintenance. Dr. Fu is a member of the Transportation Research Board's Paratransit Committee, the Editorial Advisory Board of the Journal of Transportation Research, the Intelligent Transportation Systems Society of Canada, Canadian Urban Transit Association, and the Institute of Transportation Engineers.

YAPING XIN (PXin@stantec.com) is a transportation engineer with Stantec Consulting Ltd. in Edmonton, Alberta. His work focuses on travel demand modeling, transit study, and traffic operation microsimulations. He received a Master of Applied Science in transportation engineering from the University of Waterloo. $\mathrm{He}$ is a member of Institute of Transportation Engineers. 